\title{
Wireless Sensor Networks to Monitoring Elderly People in Rural Areas
}

\author{
Gabriel Villarrubia, Juan F. De Paz, Fernando de la Prieta, and Antonio J. Sánchez \\ Department of Computer Science and Automation, University of Salamanca \\ Plaza de la Merced, s/n, 37008, Salamanca, Spain \\ $\{$ gvg, fcofds, fer, anto\} @usal.es
}

\begin{abstract}
Elderly residents who require personalized attention specific to their age-related needs generally inhabit rural areas. Users who are not of a very advanced age require only basic assistance, most commonly simple reminders to avoid forgetting or distractions. This article proposes the concept of a Smart City, which focuses on rural areas and incorporates a system to monitor and assist people of an advanced age who require a type of support which is usually complicated to provide in areas located far from urban centers. The proposed system incorporates WiFi networks, beacons, set topboxes and virtual agent organizations, and uses applications, interactive TV programs and a Wi-Fi based tracking system to monitor patients.
\end{abstract}

Keywords: Multiagent systems, wireless sensor network, health care.

\section{Introduction}

Rural areas are usually far from city centers and tend to be inhabited by elderly residents whose state of health may require basic monitoring and tracking [1] [17] [23]. There are certain services that provide direct communication to control centers through the use of alert buttons, thus providing communication for specific incidents[2] [3] [4] [11] [15]. Additionally, according to studies in this field [6], there have been many advances in areas such as telemedicine, the result of a continuous effort which can be observed in the evolution of certain devices [7]. Until now, almost all systems have focused primarily on a home environment, which requires bandwidth data connections that simply do not exist in many rural centers. This inexistence stimulated the need to create a system that could offer more extensive tracking of elderly residents, allowing them to easily interact with a television, and facilitate some of their daily tasks such as: making a doctor's appointment, selecting transportation time, confirming information from the local authorities, etc.

Current tracking systems used with elderly patients require the installation of sensors to monitor basic activities [2] [16] [18][38], and require communication networks to transmit information. In other cases, the requirements have more to do with monitoring aspects related to the environment[19], such as temperature [3], or those more specific to specific patient data such as oxygen level or pulse rate [4], even the 
location of the patient [5] [11]. These systems tend to focus on specific medical aspects and require a very specific type of device [4], often influencing the normal behavior of the user. The technology used to analyze the information varies, although systems tend to focus on the use of data mining techniques [21][26][28][29] or artificial intelligence [31][35][40] such as neural networks [8][24][25][32][41] or multiagent systems [9][20][30] [34][37][42].

Commercial systems require the installation of a large number of systems, which is costly and often a prohibitive factor in their use. This research group has already performed studies in assisting the elderly [11] [14]. The article proposes a system that integrates WiFi networks deployed in rural areas and facilitates communication of systems which are of low cost to the user since they do not require a private connection. A tracking and control system is created over the WiFi network by integrating a WiFi tag or mobile telephone to locate users in their homes and in specific nearby areas which they commonly frequent. An intelligent system with personalized multimedia content is used to control the user; this is done through a topbox which is connected to a television and offers continuous monitoring of the users in their homes to assist, for example, in reminding the patient to take pills, alerts to close an open window, or simply ask the user if they have a specific medical problem. The virtual organization of agents integrates intelligent algorithms to track and control the patient and send an alert when the patient engages in anomalous behavior.

This article is structured as follows: section 2 includes information about the proposal and the different techniques applied to monitor users; section 3 provides a case study, and section 4 presents the results and conclusions.

\section{Proposed System}

The proposed system is composed of a virtual organization [33][39] of agents which can carry out different tasks such as the localization and monitoring of users. The system was developed over the PANGEA architecture which offers possibilities such as the inclusion of light agents in different devices, for instance a topbox set. The architecture that the platform follows can be seen in Figure 1. As shown, there are two suborganizations, one associated with localization and another with processing the information obtained from the topbox set, which is in turn combined with localization.

\subsection{Passive Localization}

Passive localization is done by using the ddwrt operating system to modify the firmware in routers. Routers scan the users associated with access points as well as those not associated. Each router retrieves the information from the RSSI level measurements and sends them to a server in charge of locating the users according to the levels detected. The process is shown in Figure 2. 


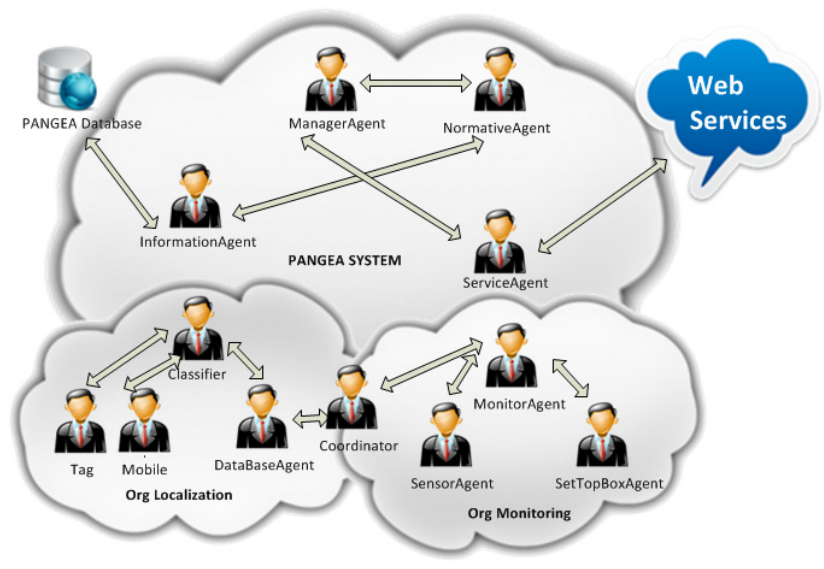

Fig. 1. Virtual organization of agents

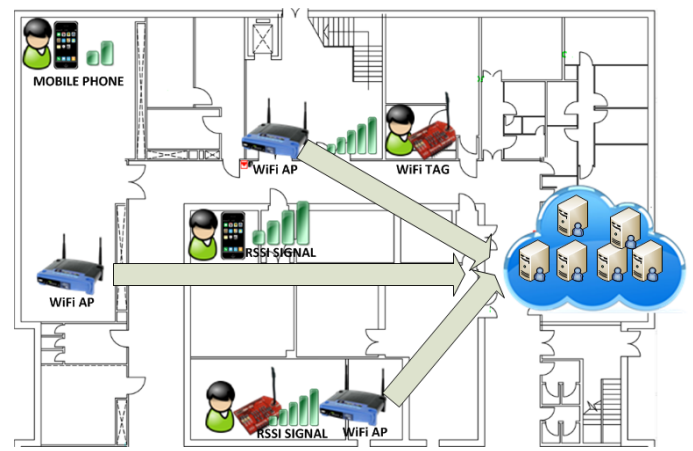

Fig. 2. Localization system architecture

In order to carry out this process, an automatic calibration is performed by a mobile phone. The mobile telephone equipped with a GPS is turned on, WiFi is activated, and position data is sent continuously to a server, which in turn cross references the position information with the RSSI level signals that were received.

Latitud, longitud, MAC BSSID antena1, RSSI antena2, ... Latitude, longitude, MAC BSSID antena1, RSSI antena2, ...

Based on this information, a training is performed by applying a SVM; the trained SVM is then used to determine the position of the user according to the signal levels detected by the WiFi antennas, without using the GPS. This makes it possible to use any WiFi device to locate users without needing to have a GPS, which in turn increases battery life. The passive localization was done by using the WiFi device seen in Figure 3. This allows for greater batter life and can be used in bracelets. Similarly, the user's mobile device can also be used. 


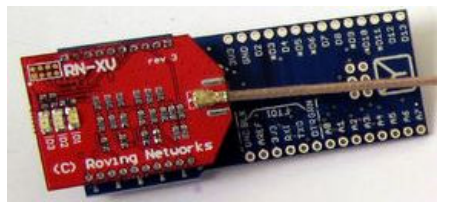

Fig. 3. User location tag

\subsection{Monitoring Users}

A topbox set with a TDT card can be used to monitor and control users through the television and still provide the user with access to regular televised broadcasts. In order to input content and facilitate interaction, the VLC was modified to display messages while watching television and capturing the interaction of users in turning on and off the device. The messages and data retrieved are listed in Table 1.

\subsection{Detecting Anomalies}

Detecting anomalous behavior in a user can be done by observing the user's behavior as they interact with the system. User interactions are grouped into categories as indicated in Table 1.

Table 1. User interactions with the system

\begin{tabular}{|l|l|}
\hline Turn on & Time \\
\hline Turn off & Time \\
\hline Pressing remote control & Time \\
\hline Interactive response & Time \\
\hline Question with alarm & Answered \\
\hline
\end{tabular}

The data gathered in Table 1 provides the information that will be used to determine normal user behavior. Three different procedures are available to determine anomalous behavior:

- Predefined rules: a rules system based on drools makes it possible to predefine rules according to the data registered in the data base. The rules are defined according to a set of conditions that when true will result in the execution of specific predefined actions.

- Interval-based: CBR [22][26][36] is used to determine a confidence interval for the values that have been detected so that the detection of a value outside the range will initiate an alert.

- Classifier: An SVM is executed within a CBR according to the cases considered anomalous and normal; each situation that is detected is classified as one of these two cases. 
The predefined rules are shown in Figure 4. As we can see, they are simple and identify a condition and an action that is executed when a given condition is true. The rules engine probes the rules and activates them automatically when the condition is true. Additionally, it can modify the rules in execution time.

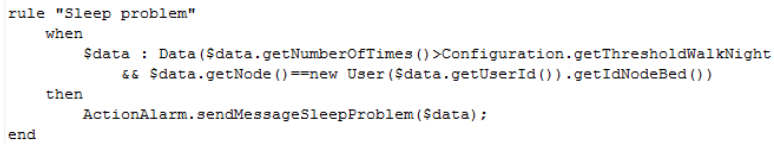

Fig. 4. Rules to determine unusual user behavior

The process for determining the confidence interval consists of grouping the days of the week by similarity and then creating confidence intervals. The definition of the cases are established as indicated in Table 2.

Table 2. Definition of a confidence interval

\begin{tabular}{|l|l|}
\hline Turned on & Length of time turned on during interval \\
\hline Number of times turned on & Number of times topbox set is turned on \\
\hline Number of times turned off & Number of times topbox set is turned off \\
\hline Remote control pulses & Number of remote control pulses during interval \\
\hline Interactive pulses & Number of interactive pulses with remote control during interval \\
\hline Alarm pulses & Number of pulses with alarm \\
\hline Day of the week & Day of the week \\
\hline Holiday & Yes or no \\
\hline Time frame & Day Schedule interval \\
\hline
\end{tabular}

A two-way ANOVA with repetition is used to group the days of the week. The two factors are the day of the week and the time interval. The model that must be followed is shown in (1).

$$
y_{i j k}=\mu+\alpha_{i}+\beta_{j}+(\alpha \beta)_{i j}+e_{i j k}
$$

A day is divided into 24 intervals of one hour. For each group the days considered different, a confidence interval is calculated for every time interval. The complete process is described in the algorithm shown in Figure 5.

The set of rules based on confidence intervals is analyzed each time new data is received from either those shown in Table 1 or at the end of each time interval. In this case, the intervals are established as one hour.

Finally, in order to obtain a more advanced procedure to determine unusual user behavior, any anomalous behavior engaged in by the user is registered in the data base. This data base stores the information referring to the information shown in Table 3. Based on this information, an LMT [13] is trained to be able to detect anomalous behavior. When the probability of an anomaly is greater than a determined threshold, the user's state will be validated. 


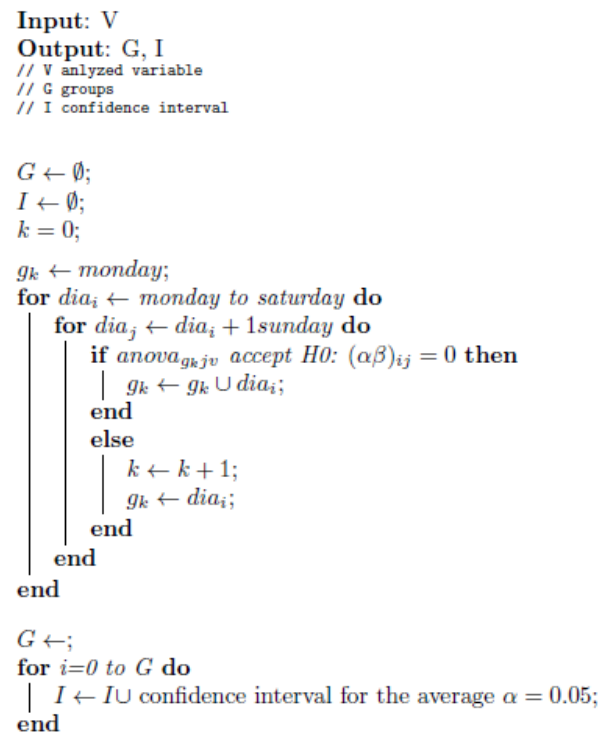

Fig. 5. Calculation of confidence intervals

Table 3. User interactions with the system

\begin{tabular}{|l|l|}
\hline Location & Zone (nearest antenna) \\
\hline Signal level & Signal level from nearest antenna \\
\hline Fields, Table 2 & $\ldots$ \\
\hline State of Alarm & Activate or Deactivate \\
\hline
\end{tabular}

\section{Case Study}

The system was tested in a small locality in the province of Salamanca (Spain). The WiFi networks were deployed using $120^{\circ}$ sector panel antennas placed in blocks of 3 to cover the full $360^{\circ}$ and provide full coverage; one of these antennas was placed in the center of the locality. For more distant areas, a sectorial antenna was used as it permits a client connection with the antenna base. The antenna used a repeater to create a virtual $\mathrm{WiFi}$ and extend coverage and by so doing take full advantage of the infrastructure without requiring the installation of many station bases. Point to point links were created with planar WiFi antennas; the signal was then replicated with sectorial antennas in all directions. The bandwidth was limited to $256 \mathrm{~Kb}$ per user due to existing legal restrictions. The tracking system was put activated in 4 homes equipped with a topbox set and a tracking tag in each one. Two mobile phones and 1 user tag were used during the calibration phase. 


\section{$4 \quad$ Results and Conclusions}

In order to analyze the functioning of the system, the localization and monitoring parts were analyzed separately. The functioning of the passive localization was analyzed first. In order to perform the calibration, the exteriors and the interiors were calibrated. Once all of the $\mathrm{WiFi}$ antennas were operative, the exterior calibration involved the use of a mobile terminal with an active GPS and WiFi, which continuously scan the WiFi networks that have been detected at that particular point, and stores the relationship of the WiFi networks with latitude and longitude. The interior calibration was done by using the blueprints of the building located in the mobile. The WiFi networks perform a scan and the user indicates the location on the plans. As the number of access points is low, the precision was set to the room level, where only one position is indicated for each room. Figure 6 shows an image of the mobile calibration system which is performed with the mobile device.

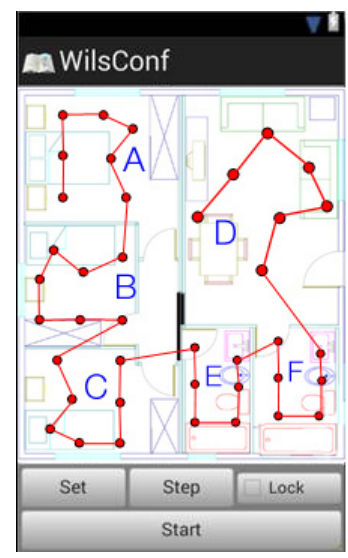

Fig. 6. Screen shot of the calibration application

The precision within one house was analyzed for a one story $85 \mathrm{~m}^{2}$ house with 3 bedrooms, 2 bathrooms and a living room. The calibration included several points within each room with approximately 15 measurements taken for each point, following the diagram shown in Figure 6. The total number of points taken was 40: 7 points per room and living room and 6 points per bathroom. An additional 20 measurements were then randomly taken for each room, living room and bathroom, for a total of 120 measurements that were subsequently classified to determine whether the room had been correctly calculated. The number of networks on each floor varied between 4 and 5. Table 4 shows the number of correct estimates for some of the different techniques that were tested. 
Table 4. Rate of accuracy for rooms detected

\begin{tabular}{|l|l|}
\hline \multicolumn{1}{|c|}{ Technique } & Correct estimates \\
\hline SVM & 112 \\
\hline J48 & 105 \\
\hline MLP & 106 \\
\hline LMT & 98 \\
\hline KNN & 87 \\
\hline
\end{tabular}

An analysis of the battery concluded that it had a life of 37 days, which included refreshing user position every 5 minutes. Battery consumption with the tag in sleep mode is $0.3 \mathrm{~mA}$, and $35 \mathrm{~mA}$ on standby.

An analysis of the SVM confusion matrix showed that the errors are mainly associated with adjacent bedrooms, which logically explains the results obtained.

The error obtained from the classification process in the town is more difficult to analyze; this is because the GPS already introduces certain errors during the measurement process, and it is complicated to apply the same indoor calibration process to the exterior process because of the time involved. The interquartile range, not related to the GPS provided position, varies between 20 and 50 meters.

In order to analyze the functioning of the monitoring system, it was first necessary to analyze the functioning of the confidence intervals and the classifier. Due to the scarce number of anomalous behavior during this initial phase, it is difficult to analyze the behavior of the system. During the evaluation process the only anomalous cases were manually generated with the specific purpose of analyzing the system. The cases were generated by varying the usual times during which the user had any interaction, and classifying these cases as anomalous. The performance of the different techniques was analyzed using the ROC curves. The same threshold of 0.3 was applied to each of the classifiers. A probability greater than 0.3 was classified as an anomalous situation. As shown in table 5, the result obtained in the area under de ROC curve is greater for LMS than for the other classifiers; nevertheless, it is necessary to increase the number of cases to determine whether the result is satisfactory.

The created system makes it easy to monitor users at a low cost since it is not necessary to install complex hardware, and because the hardware installed has other uses beyond monitoring. The battery life is sufficient enough to suggest its use as an alternative to mobiles, although it would be necessary to find more alternatives to

Table 5. Rate of accuracy for rooms detected

\begin{tabular}{|l|l|}
\hline \multicolumn{1}{|c|}{ Technique } & Correct estimates \\
\hline SVM & 0.84 \\
\hline J48 & 0.79 \\
\hline MLP & 0.88 \\
\hline LMT & 0.91 \\
\hline KNN & 0.76 \\
\hline
\end{tabular}


prolong battery life, such as installing a motion detector that would only activate the tag when it detects movement. This type of motion detector is already considered in the tag, which includes the required ports. With regard to the monitoring system, it would only be necessary to test it with more real data and load the case memory in the CBR system with real anomalous and non-anomalous cases in order to better analyze the functioning of the system.

Acknowledgments. This work has been supported by International Research Staff Exchange Scheme call FP7-PEOPLE-2012-IRSES, PIRSES-GA-2012-318878.

\section{References}

[1] Tusell, F.: Testing for interaction in two-way ANOVA tables with no replication. Computational Statistics \& Data Analysis 10(1), 29-45 (1990)

[2] Suryadevara, N.K., Gaddam, A., Rayudu, R.K., Mukhopadhyay, S.C.: Wireless Sensors Network Based Safe Home to Care Elderly People: Behaviour Detection. Procedia Engineering 25, 96-99 (2011)

[3] Noguchi, H., Mori, T., Sato, T.: Construction of network system and the first step of summarization for human daily action in the sensing room. In: Proceedings of the IEEE Workshop on Knowledge Media Networking (KMN 2002) (2002)

[4] Williams, G., Doughty, K., Bradley, D.A.: A systems approach to achieving CarerNetan integrated and intelligent telecare system. IEEE Trans. Inform. Technol. Biomed. 2(1), 1-9 (1998)

[5] Helal, S., Winkler, B., Lee, K.Y., Ran, L., Giraldo, C., Kuchibhotla, S., Mann, W.: Enabling location-aware pervasive computing applications for the elderly. In: Proceedins of IEEE 1st Conference PerCom 2003 (2003)

[6] Chan, M., Estève, S., Escriba, C., Campo, E.: A review of smart homes-Present state and future challenges 91(1), 55-81 (2008)

[7] Stowe, S., Harding, S.: Telecare, telehealth and telemedicine. European Geriatric Medicine 1(3), 193-197 (2010)

[8] Fourty, N., Guiraud, D., Fraisse, P., Perolle, G., Etxeberria, I., Val, T.: Embedded system used for classifying motor activities of elderly and disabled people. Computers \& Industrial Engineering 57(1), 419-432 (2009)

[9] Lesser, V., Atighetchi, M., Benyo, B., Horling, B., Raja, A., Vincent, R., Wagner, T., Xuan, P., Zhang, S.X.Q.: The intelligent home testbed. In: Proceedings of Autonomy Control Software Workshop, vol. 8 (1999)

[10] Pang, S., Ban, T., Kadobayashi, Y., Kasabov, N.: Personalized Mode Transductive Spanning SVM Classification Tree. Information Sciences 181, 2071-2085 (2011)

[11] Corchado, J.M., Bajo, J., Abraham, A.: GERAmI: Improving the delivery of health care in geriatric residences. IEEE Intelligent Systems (2), 19-25

[12] Zato, C., et al.: PANGEA - Platform for Automatic coNstruction of orGanizations of intElligent Agents. In: Omatu, S., Paz Santana, J.F., González, S.R., Molina, J.M., Bernardos, A.M., Rodríguez, J.M.C. (eds.) Distributed Computing and Artificial Intelligence. AISC, vol. 151, pp. 229-240. Springer, Heidelberg (2012)

[13] Landwehr, N., Hall, M., Frank, E.: Logistic Model Trees. Machine Learning 95(1-2), 161-205 (2005) 
[14] Tapia, D.I., Alonso, R.S., De Paz, J.F., Zato, C., De la Prieta, F.: International Journal of Artificial Intelligence 6(S11) (2011)

[15] Venturini, V., Carbo, J., Molina, J.M.: Methodological design and comparative evaluation of a MAS providing AmI. Expert Systems with Applications International Journal 39, 10656-10673 (2012)

[16] Sánchez-Pi, N., Carbó, J., Molina, J.M.: A Knowledge-Based System Approach for a Context-Aware System. Knowledge-Based Systems 27, 1-17 (2012)

[17] Gómez, J., Patricio, M.A., García, J., Molina, J.M.: Communication in distributed tracking systems: an ontology-based approach to improve cooperation. Expert Systems 28(4), 288-305 (2011)

[18] Serrano, E., Gómez-Sanz, J.J., Botía, A.A., Pavón, J.: Intelligent data analysis applied to debug complex software systems. Neurocomputing 72(13), 2785-2795 (2009)

[19] Fuentes-Fernández, R., Gómez-Sanz, J.J., Pavón, J.: Understanding the human context in requirements elicitation. Requirements Engineering 15(3), 267-283 (2010)

[20] Fuentes-Fernandez, R., Gomez-Sanz, J.J., Pavon, J.: Model integration in agent-oriented development. International Journal of Agent-Oriented Software Engineering 1(1), 2-27 (2007)

[21] Corchado, J.M., Fyfe, C.: Unsupervised neural method for temperature forecasting. Artificial Intelligence in Engineering 13(4), 351-357 (1999)

[22] Fdez-Riverola, F., Corchado, J.M.: CBR based system for forecasting red tides. Knowledge-Based Systems 16(5), 321-328 (2003)

[23] Tapia, D.I., Abraham, A., Corchado, J.M., Alonso, R.S.: Agents and ambient intelligence: case studies. Journal of Ambient Intelligence and Humanized Computing 1(2), 85-93 (2010)

[24] Corchado, J.M., Lees, B.: Adaptation of cases for case based forecasting with neural network support. In: Soft Computing in Case Based Reasoning, pp. 293-319 (2001)

[25] Corchado, J.M.: Redes Neuronales Artificiales: un enfoque práctico. Servicio de Publicacións da Universidade de Vigo, Vigo (2000)

[26] Bajo, J., Corchado, J.M.: Evaluation and monitoring of the air-sea interaction using a CBR-Agents approach. In: Muñoz-Ávila, H., Ricci, F. (eds.) ICCBR 2005. LNCS (LNAI), vol. 3620, pp. 50-62. Springer, Heidelberg (2005)

[27] Fraile, J.A., Bajo, J., Corchado, J.M., Abraham, A.: Applying wearable solutions in dependent environments. IEEE Transactions on Information Technology in Biomedicine 14(6), 1459-1467 (2011)

[28] Corchado, J.M., De Paz, J.F., Rodríguez, S., Bajo, J.: Model of experts for decision support in the diagnosis of leukemia patients. Artificial Intelligence in Medicine 46(3), 179200 (2009)

[29] De Paz, J.F., Rodríguez, S., Bajo, J., Corchado, J.M.: Case-based reasoning as a decision support system for cancer diagnosis: A case study. International Journal of Hybrid Intelligent Systems 6(2), 97-110 (2009)

[30] Tapia, D.I., Rodríguez, S., Bajo, J., Corchado, J.M.: FUSION@, a SOA-based multi-agent architecture. In: International Symposium on Distributed Computing and Artificial Intelligence, pp. 99-107 (2008)

[31] Corchado, J.M., Aiken, J.: Hybrid artificial intelligence methods in oceanographic forecast models. IEEE Transactions on Systems, Man, and Cybernetics, Part C: Applications and Reviews 32(4), 307-313 (2002)

[32] Corchado, J.M., Aiken, J., Rees, N.: Artificial intelligence models for oceanographic forecasting. Plymouth Marine Laboratory (2001) 
[33] Rodríguez, S., Pérez-Lancho, B., De Paz, J.F., Bajo, J., Corchado, J.M.: Ovamah: Multiagent-based adaptive virtual organizations. In: 12th International Conference on Information Fusion, FUSION 2009, pp. 990-997 (2009)

[34] Tapia, D.I., De Paz, J.F., Rodríguez, S., Bajo, J., Corchado, J.M.: Multi-agent system for security control on industrial environments. International Transactions on System Science and Applications Journal 4(3), 222-226 (2008)

[35] Borrajo, M.L., Baruque, B., Corchado, E., Bajo, J., Corchado, J.M.: Hybrid neural intelligent system to predict business failure in small-to-medium-size enterprises. International Journal of Neural Systems 21(4), 277-296 (2011)

[36] De Paz, J.F., Rodríguez, S., Bajo, J., Corchado, J.M.: Mathematical model for dynamic case-based planning. International Journal of Computer Mathematics 86(10-11), 17191730 (2009)

[37] Bajo, J., De Paz, J.F., Rodríguez, S., González, A.: Multi-agent system to monitor oceanic environments. Integrated Computer-Aided Engineering 17(2), 131-144 (2010)

[38] Tapia, D.I., Alonso, R.S., De Paz, J.F., Corchado, J.M.: Introducing a distributed architecture for heterogeneous wireless sensor networks. In: Omatu, S., Rocha, M.P., Bravo, J., Fernández, F., Corchado, E., Bustillo, A., Corchado, J.M. (eds.) IWANN 2009, Part II. LNCS, vol. 5518, pp. 116-123. Springer, Heidelberg (2009)

[39] Rodríguez, S., de Paz, Y., Bajo, J., Corchado, J.M.: Social-based planning model for multiagent systems. Expert Systems with Applications 38(10), 13005-13023 (2011)

[40] Pinzón, C.I., Bajo, J., De Paz, J.F., Corchado, J.M.S.-M.: An adaptive hierarchical distributed multi-agent architecture for blocking malicious SOAP messages within Web Services environments. Expert Systems with Applications 38(5), 5486-5499 (2011)

[41] Corchado, J.M., Bajo, J., De Paz, J.F., Rodríguez, S.: An execution time neural-CBR guidance assistant. Neurocomputing 72(13), 2743-2753 (2009)

[42] Pavon, J., Sansores, C., Gomez-Sanz, J.J.: Modelling and simulation of social systems with INGENIAS. International Journal of Agent-Oriented Software Engineering 2(2), 196-221 (2008) 